\title{
BMJ Open Quality Early prescribing outcomes after exporting the EQUIPPED medication safety improvement programme
}

Camille P Vaughan, ${ }^{1,2}$ Ula Hwang, ${ }^{3,4}$ Ann E Vandenberg, ${ }^{1}$ Traci Leong, ${ }^{5}$ Daniel Wu, ${ }^{1}$ Melissa B Stevens, ${ }^{1,2}$ Carolyn Clevenger, ${ }^{6}$ Stephanie Eucker, ${ }^{7}$ Nick Genes, ${ }^{8}$ Wennie Huang, ${ }^{7}$ Edidiong Ikpe-Ekpo, ${ }^{9}$ Denise Nassisi, ${ }^{8}$ Laura Previl, ${ }^{7}$ Sandra Rodriguez, ${ }^{10}$ Martine Sanon, ${ }^{8}$ David Schlientz, ${ }^{7}$ Debbie Vigliotti, ${ }^{11}$ S Nicole Hastings ${ }^{7,12}$

To cite: Vaughan $\mathrm{CP}$, Hwang U, Vandenberg AE, et al. Early prescribing outcomes after exporting the EQUIPPED medication safety improvement programme. BMJ Open Quality 2021;10:e001369. doi:10.1136/ bmjoq-2021-001369

Received 25 January 2021 Accepted 22 October 2021

Check for updates

(C) Author(s) (or their employer(s)) 2021. Re-use permitted under CC BY-NC. No commercial re-use. See rights and permissions. Published by BMJ.

For numbered affiliations see end of article.

Correspondence to Camille P Vaughan; camille.vaughan@emory.edu

\section{ABSTRACT}

Enhancing quality of prescribing practices for older adults discharged from the Emergency Department (EQUIPPED) aims to reduce the monthly proportion of potentially inappropriate medications (PIMs) prescribed to older adults discharged from the ED to $5 \%$ or less. We describe prescribing outcomes at three academic health systems adapting and sequentially implementing the EQUIPPED medication safety programme.

EQUIPPED was adapted from a model developed in the Veterans Health Administration (VA) and sequentially implemented in one academic health system per year over a 3-year period. The monthly proportion of PIMs, as defined by the 2015 American Geriatrics Beers Criteria, of all medications prescribed to adults aged 65 years and older at discharge was assessed for 6 months preimplementation until 12 months postimplementation using a generalised linear time series model with a Poisson distribution.

The EQUIPPED programme was translated from the VA health system and its electronic medical record into three health systems each using a version of the Epic electronic medical record. Adaptation occurred through local modification of order sets and in the generation and delivery of provider prescribing reports by local champions. Baseline monthly PIM proportions 6 months prior to implementation at the three sites were 5.6\% $(95 \%$ Cl $5.0 \%$ to $6.3 \%$ ), $5.8 \%$ (95\% Cl 5.0\% to $6.6 \%$ ) and $7.3 \%$ (95\% Cl 6.4\% to $9.2 \%$ ), respectively. Evaluation of monthly prescribing including the twelve months post-EQUIPPED implementation demonstrated significant reduction in PIMs at one of the three sites. In exploratory analyses, the proportion of benzodiazepine prescriptions decreased across all sites from approximately $17 \%$ of PIMs at baseline to $9.5 \%-12 \%$ postimplementation, although not all reached statistical significance.

EQUIPPED is feasible to implement outside the VA system. While the impact of the EQUIPPED model may vary across different health systems, results from this initial translation suggest significant reduction in specific high-risk drug classes may be an appropriate target for improvement at sites with relatively low baseline PIM prescribing rates.

\section{PROBLEM}

Enhancing quality of prescribing practices for older adults discharged from the Emergency Department (ED) (EQUIPPED)) is a medication safety programme targeting provider prescribing for older adults at the time of ED discharge. EQUIPPED has been implemented in multiple Veterans Health Administration (VA) EDs in the USA and aims to reduce the use of potentially inappropriate medications (PIMs) according to the American Geriatrics Society (AGS) Beers Criteria to $5 \%$ or less per month. ${ }^{12}$ EQUIPPED implementation involves three core components: (1) provider education provided by an on-site champion; (2) discharge medication order sets implemented through electronic health record (EHR) clinical decision support and (3) provider audit and feedback with peer benchmarking delivered through academic detailing. $^{3}$

\section{BACKGROUND}

Results from the initial EQUIPPED VA sites demonstrated reduction in the use of PIMs within and beyond the initial 12 months provider feedback period suggesting a sustained impact on provider prescribing behaviour. ${ }^{2}$ As an integrated health system with a shared EHR, national drug formulary, and common clinical data warehouse infrastructure, clinical decision support programmes promoting medication safety such as EQUIPPED may be easier to implement within VA. ${ }^{45}$ However, to impact agefriendly healthcare for older adults in the USA broadly, it is essential for clinical models to be both adaptable and effective across a variety of health systems. ${ }^{6} 7$ Based on promising early results, the EQUIPPED programme was exported from the VA to three academic health systems affiliated with VA EQUIPPED 
sites. Adaptation outside of the VA occurred through a sequential implementation strategy allowing for lessons learnt to be applied to subsequent sites. Here, we describe adaptation of EQUIPPED from VA to non-VA academic health centres and early prescribing outcomes at each site.

\section{MEASUREMENT}

The primary effectiveness outcome was the monthly PIM proportion (\# PIMs/all medications prescribed to adults aged 65 and older at ED discharge). Similar to previous experiences implementing EQUIPPED, a 6-month period before any EQUIPPED activities began defined the baseline prescribing period. The implementation period differed by sites depending on the time needed to implement the order sets within the EHR. The postimplementation period was defined as the 12 months after the beginning of provider feedback.

Rate ratios and their respective $95 \%$ CIs were calculated to describe the aggregate PIM percentages in the preEQUIPPED and post-EQUIPPED periods. Generalised linear time series models assuming a Poisson distribution for the monthly PIMs rates were fitted. The total number of prescriptions served as the offset term in the model, and a piecewise, non-linear regression model was used to evaluate the pattern of PIM prescriptions over time. All models contained three basic parameters accounting for the preintervention trend (preintervention slope), the change in level at the intervention point, and the difference in trend between the two periods (change in slope from preintervention). Correlograms were used to check for autocorrelation in the residuals using the Durbin Watson test. SEs were calculated based on the Newey-West method to account for the autocorrelation. Based on observed autocorrelation, the post intervention trend was adjusted by 1-3 month lags depending on the institution. Based on an observation that the same three PIM drug classes (antihistamines, benzodiazepines, muscle relaxants) were the most frequently prescribed PIMs at each site, an exploratory analysis of class-specific change within each site was conducted. All analyses were performed using the statistical software R, V.3.0.1 (R Foundation for Statistical Computing, Vienna, Austria), and $p$ values of 0.05 or less were considered statistically significant.

\section{DESIGN}

Each implementation site included a local site champion and a multidisciplinary team including expertise in geriatric medicine, emergency medicine, quality improvement, clinical pharmacy services, and clinical data warehouse extraction. EQUIPPED was implemented sequentially across the three sites with one site implementing each year. Sequential implementation allowed subsequent sites to leverage tools and lessons learnt based on the experience of prior implementation sites. Details of this implementation strategy as a learning health system approach have been published previously. Throughout the 3-year project, site champions and evaluation team leads met twice a month to discuss progress at the implementing site and lessons learnt. During a given implementation year, the local EQUIPPED team met on a weekly basis to facilitate order set implementation, adaptation of provider feedback forms, and prepare for the local site launch.

\section{STRATEGY: ADAPTATION OF VA EQUIPPED TO ACADEMIC SITE IMPLEMENTATION}

The institutional review boards at each site determined that implementation of the EQUIPPED medication safety programme was a quality improvement activity and not human subjects research. Patients or the public were not involved in the design of this programme. EQUIPPED was developed using the Vision-Analysis -Team-Aim-MapMeasure-Change-Sustain (VA-TAMMCS) framework for quality improvement. ${ }^{3}$ During the Change step, teams used the Plan-Do-Study-Act (PDSA) model for rapid cycle change and applied single PDSA cycles to each component of the EQUIPPED programme as it was adapted for an implementation site. Because sites were sequentially adapting the VA EQUIPPED programme, lessons learnt were applied to subsequent implementation site PDSA cycles.

The first PDSA cycle involved adapting the EQUIPPED order sets implemented at the initial VA site. The initial VA order set templates included treatment suggestions based on the VA formulary for common conditions leading to discharge from the ED for older adults. Adaptations included tailoring medication selections, such as different formulary preferences or antibiotic options in consultation with local antibiotic stewardship experts, and determining options based on site specific factors (eg, different clinic referrals). All of the sites engaged in this implementation were part of health systems with the Epic EHR. After the first site determined the optimal location for order set placement within discharge workflows to easily facilitate provider use, the second and third sites adopted a similar strategy.

The second PDSA cycles both within and across sites involved optimisation of provider feedback reports. Unlike VA sites, which were eventually able to leverage the VA's centralised clinical data warehouse infrastructure to develop an electronic PIM dashboard for any VA $\mathrm{ED},{ }^{9}$ each non-VA site worked with local data extraction services to optimise collection of monthly prescribing data for provider feedback reports. A tool developed within the VA clinical data warehouse to identify PIMs according to the AGS Beers Criteria category 1 medications that are always considered potentially inappropriate in older adults was adapted for use by the three sites. During the initial development and implementation of EQUIPPED at VA sites, the EQUIPPED team determined that some Beers category 1 PIMs with conditions requiring chart review, such as proton pump inhibitors and antipsychotic medication, were typically prescribed appropriately in 
the ED setting. Thus, these medication classes were not flagged as inappropriate for provider feedback reports. During this second PDSA cycle for non-VA sites, chart review of 1-3 months of encounters with proton pump inhibitor or antipsychotic prescriptions at discharge were reviewed at each site. This chart review revealed the same pattern of appropriate prescribing in non-VA EDs, and so proton pump inhibitors and antipsychotic medications were not included in provider feedback reports. The initial site received data through the health system's pharmacy service, which then required processing by an EQUIPPED team member to create provider reports. The second site modified the VA tool to develop a pivot table in Excel with Visual Basic programming and then Tableau as a data visualisation tool to create provider reports more quickly. The use of Tableau was also implemented by the third site.

Implementation of provider feedback occurred through a third PDSA cycle. Provider feedback at VA sites involved an initial one-on-one session with a local EQUIPPED champion. Earlier VA ED sites included a staff provider group of 8-20 providers (including MDs and advanced practice providers (APPs)). Two of the three non-VA academic health system sites employed larger ED provider groups with 70-80 MDs and APPs so that providing an initial one-on-one feedback session in a single month was impractical. At the first site, the implementation team conducted the one-on-one session with a provider when they received their first report with a PIM prescription. This adaptation lead to a majority of providers receiving a report within the first 3 months of the postimplementation period. The second site implemented one-on-one feedback with three different EQUIPPED champions delivering feedback. The third site had a smaller provider group and was able to reach all providers with at least one initial in-person feedback session. The third site also conducted a subsequent in-person feedback session with providers who continued prescribing PIMs above the threshold of $5 \%$ per month.

\section{RESULTS}

Site characteristics of the three implementation sites are described in figure 1, and additional details have been published previously. ${ }^{8}$ Two of the sites were level 1 trauma centres and the third a level 3 trauma centre. Annual ED encounters ranged from approximately 80000-140000 with $15 \%-20 \%$ of the patients seen classified as 65 years of age and older across the sites. Prescribers at each site included attending MDs, APPs and resident physicians. Two sites also had pharmacists in the ED. One of the EDs was accredited as a Level 1 Geriatric ED at the time of implementation. The other two sites had existing EHRbased alerts that activated as an on-screen alert if providers selected certain PIMs.

At site 1 , the time series shown in figure 1 exhibited a negative trend with the solid line representing the fit of the time series and the dotted line with the associated 95\% CI. The corresponding aggregate PIM percentages were $5.6 \%$ (95\% CI $5.0 \%$ to $6.3 \%$ ) for the pre-EQUIPPED period and $5.1 \%(95 \%$ CI $4.7 \%$ to $5.5 \%)$ for the postEQUIPPED period (table 1). At site 2, the trend in
Site 1

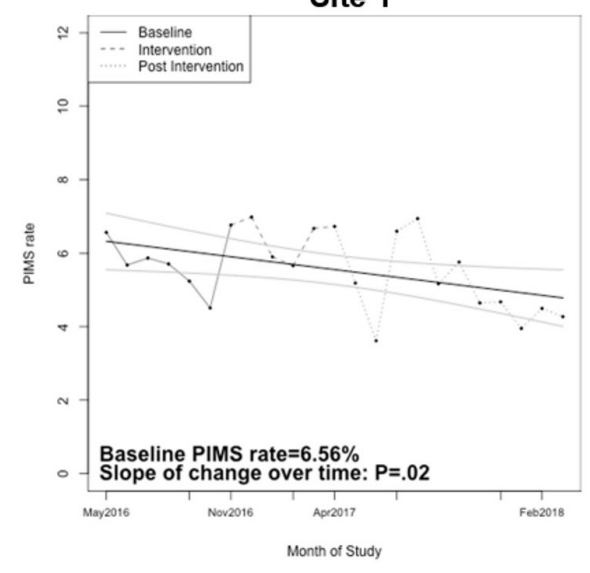

139,728 ED encounters; $15 \%$ geriatric

53 Attendings
62 Residents
11 Physician assistants
27 Nurse practitioners
5 Pharmacists
1 Site providers delivering feedback

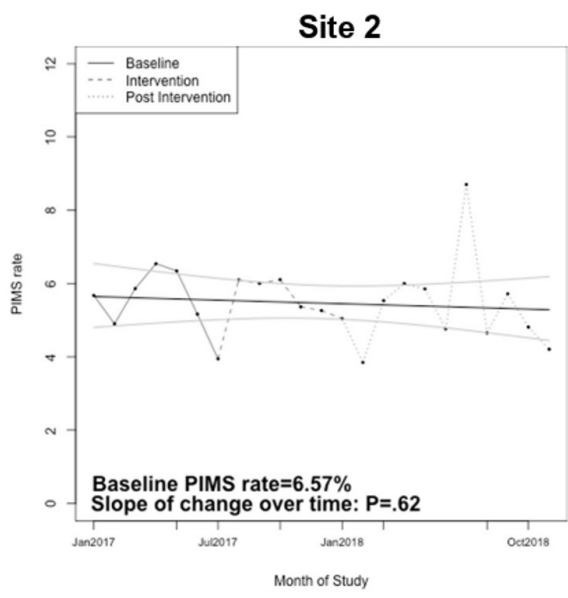

110,593 ED encounters; $19.1 \%$ geriatric

32 Attendings
67 Residents
26 Physician assistants
2 Nurse practitioners
0 Pharmacists
3 Site providers delivering feedback

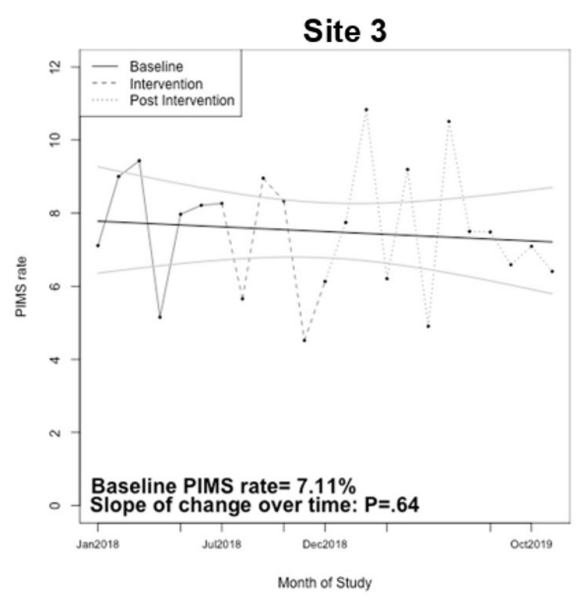

79,258 ED encounters; $21.2 \%$ geriatric

38 Attendings
31 Residents
13 Physician assistants
0 Nurse practitioners
1 Pharmacist

2 Site providers delivering feedback

Figure 1 General time series analysis of potentially inappropriate medication (PIMs) prescribed, with implementation year site characteristics. ED, emergency department. 
Table 1 Aggregate pre-EQUIPPED and post-EQUIPPED PIM prescribing and specific PIM drug classes at each implementation site

$\begin{array}{ll}\text { Pre-EQUIPPED (\%) } & \text { Post-EQUIPPED (\%) } \\ (95 \% \text { Cl for All PIMs) } & (95 \% \text { Cl for All PIMs) }\end{array}$

\begin{tabular}{|c|c|c|c|}
\hline \multicolumn{4}{|l|}{ Site 1} \\
\hline All PIMs & 5.6 (5.0 to 6.3 ) & 5.1 (4.7 to 5.5$)$ & 0.02 \\
\hline Benzodiazepine & 16.6 & 9.5 & 0.04 \\
\hline Skeletal muscle relaxant & 34.4 & 36.9 & 0.44 \\
\hline Antihistamine & 15.8 & 13.4 & 0.15 \\
\hline \multicolumn{4}{|l|}{ Site 2} \\
\hline All PIMs & 5.8 (5.0 to 6.6 ) & 5.4 (4.8 to 6.0$)$ & 0.62 \\
\hline Benzodiazepine & 16.9 & 10.0 & 0.09 \\
\hline Skeletal muscle relaxant & 21.9 & 21.3 & 0.84 \\
\hline Antihistamine & 49.3 & 49.2 & 0.57 \\
\hline \multicolumn{4}{|l|}{ Site 3} \\
\hline All PIMs & 7.3 (6.4 to 9.2$)$ & 7.5 (6.6 to 8.4$)$ & 0.64 \\
\hline Benzodiazepine & 17.3 & 12.0 & 0.05 \\
\hline Skeletal muscle relaxant & 24.5 & 14.5 & 0.04 \\
\hline Antihistamine & 38.2 & 43.2 & 0.52 \\
\hline
\end{tabular}

${ }^{*}$ Percentages for specific PIM classes represent the \% of that class among all PIM prescriptions.

$\dagger P$ value represents general time series model assuming a Poisson distribution.

EQUIPPED, Enhancing quality of prescribing practices for older adults discharged from the Emergency Department; PIMs, potentially inappropriate medications.

PIM prescribing over time did not change significantly $(\mathrm{p}=0.62)$ (figure 1$)$. The corresponding aggregate PIM percentages were $5.8 \%$ (95\% CI $5.0 \%$ to $6.6 \%$ ) for the pre-EQUIPPED period and $5.4 \%$ (95\% CI $4.8 \%$ to $6.0 \%)$ for the post-EQUIPPED period (table 1). At site 3, the time trend in PIM prescribing did not change significantly $(\mathrm{p}=0.64)$ (figure 1$)$. The corresponding aggregate PIM percentages were $7.3 \%$ (95\% CI $6.4 \%$ to $9.2 \%$ ) for the pre-EQUIPPED period and $7.5 \%(95 \%$ CI $6.6 \%$ to $8.4 \%$ ) for the post-EQUIPPED period (table 1 ).

Table 1 shows additional detail regarding the exploratory analysis of specific PIM drug classes across the three sites. The proportion of benzodiazepine prescriptions decreased from approximately $17 \%$ of PIMs at baseline to $9.5 \%-12 \%$ postimplementation. Additionally, at site 3 , the proportion of musculoskeletal relaxants decreased significantly compared with baseline, decreasing from $24.5 \%$ to $14.5 \%$ (table 1). Antihistamine prescriptions did not change significantly at any of the sites.

\section{LESSONS AND LIMITATIONS}

All three sites successfully implemented the core components of the EQUIPPED medication safety programme. Although statistically significant reductions in PIMS prescribing rates were not seen consistently across the three implementation sites, and no site decreased the overall PIM prescribing percentage to less than $5 \%$ in the first 12 months after implementation, reductions were observed in specific high-risk drug classes. These results contrast with previous VA EQUIPPED analyses using the same methods, in which all four initial VA sites showed a significant and sustained reduction in overall use of PIMs and other studies indicating positive effects of clinical decision support to influence ED provider

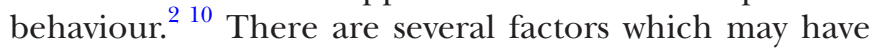
influenced these early results and will inform next steps in understanding best practices for implementing the EQUIPPED programme.

In this initial export of the EQUIPPED programme, sites were selected based on champions at each site with previous VA EQUIPPED implementation experience and a common EHR at all sites, facilitating implementation of the clinical decision support tools. The selected sites had relatively low baseline PIM proportions when compared with the VA sites (5.64-7.3 vs $7.4 \%-11.9 \%)$. It is possible that different site selection, such as those with higher PIM rates, would have yielded different results.

Similar to VA sites, the sequential implementation approach for the academic health system sites permitted sharing of resources that facilitated implementation, particularly with regard to EQUIPPED order sets and the data collection necessary to give provider audit, feedback and peer benchmarking monthly reports. Data visualisation platforms such as Tableau also facilitated greater automation for the production of monthly provider feedback reports, which was important to promote sustainability at the site.

Whereas in the VA system a centralised EQUIPPED team was able to sustain reporting of PIM prescriptions as needed for multiple implementation sites through 
the VA's clinical data warehouse, each academic health system site needed to identify a local data extraction team for ongoing evaluation of ED PIM prescribing. Thus, we report here a relatively short postimplementation period of 12 months, which was determined by the initial funding cycle vs a longer 25-45 months reported at VA sites. Sustaining ongoing evaluation of ED PIM rates at the academic health system sites is more dependent on local health system priorities and resources, which limited the availability of longer term follow-up at the three sites. Longer-term follow-up would also facilitate evaluation of the impact of a medication safety programme like EQUIPPED on relevant patient outcomes such as repeat visits the to the $\mathrm{ED}$, subsequent hospitalisation and adverse drug events.

Despite not observing consistently an overall site level reduction in PIM rates at two of the three sites, a trend towards reduced prescribing of certain high-risk drug classes, specifically benzodiazepines, was observed at all three EQUIPPED sites. At the third site, a specific focus on musculoskeletal relaxants during detailing also resulted in a significant and sustained reduction. A recent evaluation of benzodiazepine prescribing patterns in the USA from 2013 to 2017 suggests a small decline in the prescribing of benzodiazepines for older adults who receive health coverage through Medicare and that even steeper declines were observed in VA where focused programmes were implemented nationally to reduce benzodiazepine use. ${ }^{11}$ The present results suggest that among sites with baseline PIM prescribing rates that are closer to the EQUIPPED target of $5 \%$ or less, a focus on specific problematic drug classes may be a more meaningful approach.

Future evaluation will include an additional assessment of internal and external factors that may have impacted implementation effectiveness through provider surveys and implementation team focus groups. This evaluation will also include sites that are part of additional projects to spread the EQUIPPED model within and across additional academic health system and VA sites. Factors that have been identified as important in other geriatric models of care include effective clinician leadership, senior leadership champions and shifting organisational culture. $^{6}$

\section{CONCLUSION}

EQUIPPED represents a quality improvement programme to enhance safe prescribing towards older adults in the ED setting that is feasible to implement across multiple health systems. Early results after exporting EQUIPPED from the VA to three academic health systems demonstrated significant reduction in PIM prescribing at one of the sites and a trend towards reduced prescribing of specific high-risk medication classes at all sites. Achieving significant reduction in specific high-risk drug classes may be a more appropriate target for sites that have relatively low baseline PIM prescribing rates.
Author affiliations

${ }^{1}$ Medicine (CPV, AEV, MBS) \& Emergency Medicine (DW), Emory University School of Medicine, Atlanta, Georgia, USA

${ }^{2}$ Birmingham/Atlanta VA Geriatric Research Education and Clinical Center, Atlanta VA Medical Center, Decatur, Georgia, USA

${ }^{3}$ Emergency Medicine, Yale School of Medicine, New Haven, Connecticut, USA

${ }^{4}$ Geriatric Research Education and Clinical Center, James J. Peters Veterans Affairs Medical Center, Bronx, NY, USA

${ }^{5}$ Biostatistics and Bioinformatics, Emory University School of Public Health, Atlanta, Georgia, USA

${ }^{6}$ Emory University, School of Nursing, Atlanta, Georgia, USA

${ }^{7}$ Medicine (LP, DS, SNH) \& Emergency Medicine (SE, WH), Duke University School of Medicine, Durham, NC, USA

${ }^{8}$ Emergency Medicine (NG, DN) and Medicine (MS), Icahn School of Medicine at Mount Sinai, New York, New York, USA

${ }^{9}$ Emergency Medicine, The Southeast Permanente Medical Group, Atlanta, Georgia, USA

${ }^{10}$ Columbia University School of Social Work, New York, New York, USA

${ }^{11}$ Pharmacy Services, Grady Health System, Atlanta, Georgia, USA

${ }^{12}$ Centre of Innovation to Accelerate Discovery and Practice Transformation, Durham VA Health Care System, Durham, North Carolina, USA

Contributors CPV: study concept and design, acquisition of data, analysis and interpretation of data, preparation of manuscript and final approval of manuscript. UH: study concept and design, acquisition of data, analysis and interpretation of data, preparation of manuscript and final approval of manuscript. AEV: study concept and design, acquisition of data, analysis and interpretation of data, preparation of manuscript and final approval of manuscript. TL: study concept and design, analysis and interpretation of data, preparation of manuscript and final approval of manuscript, DW: acquisition of data, interpretation of data, preparation of manuscript and final approval of manuscript. MBS: study concept and design, interpretation of data, preparation of manuscript and final approval of manuscript. CC: study concept and design, interpretation of data, preparation of manuscript and final approval of manuscript. SE: acquisition of data, interpretation of data, preparation and final approval of manuscript. NG: acquisition of data, interpretation of data, preparation of manuscript and final approval of manuscript. WH: acquisition of data, interpretation of data, preparation of manuscript and final approval of manuscript. El-E: acquisition of data, interpretation of data, preparation of manuscript and final approval of manuscript. DN: acquisition of data, interpretation of data, preparation of manuscript and final approval of manuscript. LP: acquisition of data, interpretation of data, preparation of manuscript and final approval of manuscript. SR: acquisition of data, interpretation of data, preparation of manuscript and final approval of manuscript. MS: acquisition of data interpretation of data, preparation of manuscript and final approval of manuscript. DS: acquisition of data, interpretation of data, preparation of manuscript and final approval of manuscript. DV: acquisition of data, interpretation of data, preparation of manuscript and final approval of manuscript. SNH: study concept and design, acquisition of data, analysis and interpretation of data, preparation of manuscript and final approval of manuscript.

Funding Funding was provided by grant R18HS024499 (PI Vaughan) from the Agency for Healthcare Research and Quality. SNH received support from the Durham Centre of Innovation to Accelerate Discovery and Practice Transformation (ADAPT), (CIN 13-410) at the Durham VA Healthcare System.

Open access This is an open access article distributed in accordance with the Creative Commons Attribution Non Commercial (CC BY-NC 4.0) license, which permits others to distribute, remix, adapt, build upon this work non-commercially, and license their derivative works on different terms, provided the original work is properly cited, appropriate credit is given, any changes made indicated, and the use is non-commercial. See: http://creativecommons.org/licenses/by-nc/4.0/.

\section{REFERENCES}

1 By the 2019 American Geriatrics Society Beers Criteria ${ }^{\circledR}$ Update Expert Panel. American geriatrics Society 2019 updated AGS beers Criteria $®$ for potentially inappropriate medication use in older adults. J Am Geriatr Soc 2019;67:674-94.

2 Stevens M, Hastings SN, Markland AD, et al. Enhancing quality of provider practices for older adults in the emergency department (equipped). J Am Geriatr Soc 2017;65:1609-14. 
3 Stevens MB, Hastings SN, Powers J. Enhancing the quality of prescribing practices for older veterans discharged from the emergency department: preliminary results from equipped, a novel multicomponent interdisciplinary quality improvement initiative. J Am Geriatr Soc 2015;63:1025-9.

4 Burningham Z, Chen W, Sauer BC, et al. Va geriatric scholars program's impact on prescribing potentially inappropriate medications. Am J Manag Care 2019;25:425-30.

5 Vandenberg AE, Echt KV, Kemp L, et al. Academic detailing with provider audit and feedback improve prescribing quality for older veterans. J Am Geriatr Soc 2018;66:621-7.

6 Hshieh TT, Yang T, Gartaganis SL, et al. Hospital elder life program: systematic review and meta-analysis of effectiveness. Am J Geriatr Psychiatry 2018;26:1015-33.

7 Fox MT, Persaud M, Maimets I, et al. Effectiveness of acute geriatric unit care using acute care for elders components: a systematic review and meta-analysis. J Am Geriatr Soc 2012;60:2237-45.
8 Vandenberg AE, Kegler M, Hastings SN, et al. Sequential implementation of the equipped geriatric medication safety program as a learning health system. Int $J$ Qual Health Care 2020;32:470-6.

9 Burningham Z, Jackson GL, Kelleher J, et al. The enhancing quality of prescribing practices for older veterans discharged from the emergency department (equipped) potentially inappropriate medication Dashboard: a suitable alternative to the In-person academic detailing and standardized feedback reports of traditional equipped? Clin Ther 2020;42:573-82.

10 Patterson BW, Pulia MS, Ravi S, et al. Scope and influence of electronic health Record-Integrated clinical decision support in the emergency department: a systematic review. Ann Emerg Med 2019;74:285-96.

11 Maust DT, Kim HM, Wiechers IR, et al. Benzodiazepine use among Medicare, commercially insured, and veteran older adults, 20132017. J Am Geriatr Soc 2021;69:98-105. 